\title{
ON FIRST-ORDER DIFFERENTIAL OPERATORS WITH BOHR-NEUGEBAUER TYPE PROPERTY
}

\author{
ARIBINDI SATYANARAYAN RAO \\ Department of Mathematics, Concordia Univ., Montreal, P.乌uebec, Canada H3G 1M8 \\ (Received January 1, 1987 and in revised form April 20, 1987)
}

ABSTRACT. We consider a differential equation $\frac{d}{d t} u(t)-B u(t)=f(t)$, where the functions $\mathrm{u}$ and $\mathrm{f}$ map the real line into a Banach space $\mathrm{X}$ and $\mathrm{B}: \mathrm{X} \rightarrow \mathrm{X}$ is a bounded linear operator. Assuming that any Stepanov-bounded solution $u$ is Stepanov almost-periodic when $f$ is Bochner almost-periodic, we establish that any Stepanov-bounded solution $u$ is Bochner almost-periodic when $f$ is Stepanov almost-periodic. Some examples are given in which the operator $\frac{d}{d t}-B$ is shown to satisfy our assumption.

KEY WORDS AND PHRASES. Bounded linear operator, differential operator, BohrNeuqebauer property, Bochner (Stepanov) almost-periodic function. 1980 AMS SUBJECT CLASSIFICATION CODE. 34GXX, 34G10, 34C27.

\section{INTRODUCTION.}

Suppose $X$ is a Banach space and $J$ is the interval $-\infty<t<\infty$. A function $f \in L_{1 \circ c}^{p}(J ; X)$ with $1 \leqq p<\infty$ is said to be Stepanov-bounded or $S^{p}$-bounded on $J$ if

$$
\|f\|_{S^{p}}=\sup _{t \in J}\left[\begin{array}{l}
t+1 \\
t
\end{array}\|f(s)\|^{p_{d s}}\right]^{1 / p}<\infty .
$$

our first result is as follows.

THEOREM 1. Suppose $\mathrm{f}: \mathrm{J} \rightarrow \mathrm{X}$ is a continuously differentiable $\mathrm{s}^{1}$-bounded function, and $\mathrm{f}^{\prime}$ is an $\mathrm{S}^{\mathrm{p}}$-bounded function with $l \leqq p<\infty$. Then, (a) if $p=1, f$ is bounded on $\mathrm{J}$, and (b) if $\mathrm{p}>1, \mathrm{f}$ is bounded and uniformly continuous on $\mathrm{J}$.

2. PROOF OF THEOREM 1.

(a) $p=1$. For an arbitrary but fixed $t \in J$, there exists at least one point $\tau_{t} \in[t-1, t]$ such that

$$
\left\|f\left(\tau_{t}\right)\right\|=\inf _{t-1 \leqq s \leqq t}\|f(s)\|
$$

Consequently, we have

$$
\left\|f\left(\tau_{t}\right)\right\| \leqq s_{t-1}^{t}\|f(s)\| d s \leqq\|f\| s^{1} \text {, by (1.1). }
$$


Hence, from the $S^{l}$-boundedness of $f^{\prime}$, we obtain

$$
\begin{aligned}
& \|f(t)\|=\left\|f\left(\tau_{t}\right)+s_{\tau_{t}}^{t} f^{\prime}(s) d s\right\| \\
& \leqq\left\|f\left(\tau_{t}\right)\right\|+\int_{\tau_{t}}^{t}\left\|f^{\prime} \quad(s)\right\| d s \\
& \leqq\|f\|_{S^{l}}+\left\|f^{\prime}\right\| S^{l} \text {. }
\end{aligned}
$$

(b) $p>1$. By Hölder's inequality, the $s^{p}$-boundedness of $f^{\prime}$ implies the $S^{l}$-boundedness of $f^{\prime}$. Hence, as shown above, $f$ is bounded on $J$. inequality,

Moreover, for $0<t_{2}-t_{1}<1$ and $\frac{1}{p}+\frac{1}{q}=1$, we have, again by Hölder's

$$
\begin{aligned}
& \left\|f\left(t_{2}\right)-f\left(t_{1}\right)\right\|=\| s_{t_{1}}^{t_{2}} f^{\prime} \text { (s) ds } \|
\end{aligned}
$$

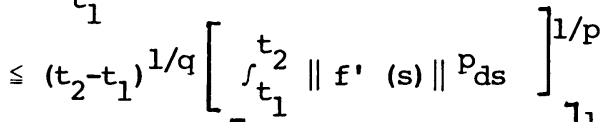

$$
\begin{aligned}
& \leqq\left(t_{2}-t_{1}\right)^{1 / q}\left[\begin{array}{c}
t_{1}+1 \\
\int_{t_{1}}
\end{array}\left\|f^{\prime}(s)\right\|^{p_{d s}}\right]^{1 / p} \\
& \leqq\left(t_{2}-t_{1}\right)^{1 / q}\left\|f^{\prime}\right\| S^{p} \text {. }
\end{aligned}
$$

Therefore $f$ is uniformly continuous on $J$, completing the proof of the theorem.

REMARK. If $\mathrm{f}: \mathrm{J} \rightarrow \mathrm{X}$ is a continuously differentiable $\mathrm{s}^{1}$-almost periodic function, with $f^{\prime}$ being $S^{p}$-bounded on $J(1<p<\infty)$, then $f$ is (uniformly) almostperiodic from $J$ to $X$ (see pp. 3 and 77, Amerio-Prouse [1] for the definitions of (uniform) almost-periodicity and $\mathrm{S}^{\mathrm{P}}$-almost periodicity).

PROOF. By Theorem 1, $\mathrm{f}$ is uniformly continuous on J. Hence, by Theorem 7, p. 78, Amerio-Prouse [1], $f$ is (uniformly) almost-periodic from $J$ to $X_{\text {. }}$

\section{MAIN RESULT.}

Let $B$ be a bounded linear operator on a Banach space $X$ into itself. Then the differential operator $\frac{d}{d t}-B$ is said to have Bohr-Neugebauer property if, for any (uniformly) almost-periodic $X$-valued function $f$, any bounded (on J) solution of the equation

$$
\frac{d}{d t} u(t)-B u(t)=f(t) \text { on } J
$$

is (uniformly) almost-periodic.

our result is as follows.

THEOREM 2. In a Banach space $X$, let the differential operator $\frac{d}{d t}-B$ be such that, for any (uniformly) almost-periodic $x$-valued function $f$, any $S^{l}$-bounded solution of the equation (3.1) is $s^{l}$-almost periodic. Then, for any $s^{l}$-almost periodic continuous $\mathrm{X}$-valued function $\mathrm{g}$, any $\mathrm{s}^{\mathrm{l}}$-bounded solution $\mathrm{u}: \mathrm{J} \rightarrow \mathrm{X}$ of the equation

$$
\frac{d}{d t} u(t)-B u(t)=g(t) \text { on } J
$$

is (uniformly) almost-periodic. 
PROOF. Since $g$ is $S^{l}$-almost periodic from $J$ to $X$, it is $S^{l}$-bounded on $J$. Consequently, $u^{\prime}=\mathrm{Bu}+\mathrm{g}$ is $\mathrm{s}^{1}$-bounded on $\mathrm{J}$. Hence, by Theorem $\mathrm{l}, \mathrm{u}$ is bounded on $\mathrm{J}$. Now consider a sequence $\left\{\phi_{n}(t)\right\}_{n=1}^{\infty}$ of non-negative continuous functions on $\mathrm{J}$ such that

$$
\phi_{n}(t)=0 \text { for }|t| \geqq n^{-1}, \int_{-n^{-1}}^{n^{-1}} \phi_{n}(t) d t=1 \text {. }
$$

The convolution of $\mathrm{u}$ and $\phi_{\mathrm{n}}$ is defined by

$$
\left(u^{\star} \phi_{n}\right)(t)=\int_{J} u(t-s) \phi_{n}(s) d s=\int_{J} u(s) \phi_{n}(t-s) d s .
$$

Then, by (3.2), we have

$$
\frac{d}{d t}\left(u^{\star} \phi_{n}\right) \quad(t)-B\left(u^{\star} \phi_{n}\right) \quad(t)=\left(g^{\star} \phi_{n}\right) \quad(t) \text { on } J .
$$

We note that

$$
\sup _{t \in J}\left\|\left(u^{*} \phi_{n}\right) \quad(t)\right\| \leqq \sup _{t \in J}\|u(t)\| \text {. }
$$

Further, we can show that $\mathrm{g}^{\star} \phi_{\mathrm{n}}$ is (uniformly) almost-periodic from $\mathrm{J}$ to $\mathrm{X}$ (see the proof of Theorem 7, p. 78, Amerio-Prouse [1]).

Therefore, by our assumption on the operator $\frac{d}{d t}-B,\left(u^{\star} \phi_{n}\right)(t)$ is $s^{l}$-almost periodic for all $\mathrm{n}=1,2, \ldots$.

By (3.2), we have the representation

$$
u(t)=u(0)+s_{0}^{t} B u(s) d s+\int_{0}^{t} g(s) d s \text { on } J .
$$

If $t_{2}>t_{1}$, then

$$
\| \int_{t_{1}}^{t_{2}} B u \text { (s) ds }\|\leqq\| B\left\|\cdot \sup _{t \in J}\right\| u(t) \| \cdot\left(t_{2}-t_{1}\right) .
$$

Hence $\int_{0}^{t} \mathrm{Bu}(\mathrm{s})$ ds is uniformly continuous on $\mathrm{J}$. Also, by Theorem 8, p. 79, AmerioProuse $[1], s_{0}^{t} g(s)$ ds is uniformly continuous on $J$. Consequently, $u$ is uniformly continuous on $\mathrm{J}$.

Similarly, from (3.5), it follows that $u^{*} \phi_{n}$ is uniformly continuous on $J$. So, by Theorem 7, p. 78, Amerio-Prouse [1], $\mathrm{u}^{*} \phi_{\mathrm{n}}$ is (uniformly) almost-periodic for all $\mathrm{n}=1,2, \ldots$.

Now, by the uniform continuity of $u$ on $J$, the sequence of convolutions $\left(u^{\star} \phi_{n}\right)$ converges to $u(t)$ uniformly on $J$. Hence $u$ is (uniformly) almost-periodic from $\mathrm{J}$ to $\mathrm{X}$, which completes the proof of the theorem.

\section{NOTES.}

(i) Suppose $X$ is a Hilbert space and $B$ is a self-adjoint bounded linear operator on $x$ into itself. Then we know that the operator $\frac{d}{d t}-B$ has Bohr-Neugebauer property (see Zaidman [4]). Given an (uniformly) almost-periodic X-valued function $f$, suppose that $u$ is an $s^{1}$-bounded solution of the equation (3.1). If we replace $q$ by $f$ in the proof of our Theorem 2, then, by the Bohr-Neugebauer property of the operator $\frac{d}{d t}-B$, it follows that $u$ is (uniformly) almost-periodic from $J$ to $x$. Thus the operator $\frac{d}{d t}-B$ satisfies the hypothesis of Theorem 2 . 
(ii) Now suppose $\mathrm{X}$ is a separable Hilbert space and B is a completely continuous normal operator on $X$ into itself. Then, by Theorem 1 of cooke [3], the operator $\frac{d}{d t}-B$ has Bohr-Neugebauer property. Consequently, the operator $\frac{d}{d t}-B$ satisfies the assumption of Theorem 2.

(iii) Finally, suppose $\mathrm{X}$ is a reflexive space and $\mathrm{B}=0$. Then the operator $\frac{d}{d t}$ has Bohr-Neugebauer property (see Amerio-Prouse [1], p. 55 and Authors' Remark on p. 82). Hence the operator $\frac{d}{d t}$ satisfies the assumption of Theorem 2 .

REFERENCES

1. AMERIO, L. and PROUSE, G.

Almost periodic functions and functional equations, Van Nostrand Reinhold Company, 1971.

2. BOCHNER, S. and NEUMANN, J.V. On compact solutions of operationaldifferential equations I, Ann, of Math., 36 (1935), 255-291.

3. COOKE, R. Almost periodicity of bounded and compact solutions of differential equations, Duke Math. J., 36 (1969), 273-276.

4. ZATDMAN, S. Quasi-periodicità per un'equazione operazionale del primo ordine, Rend. Accad. Naz. dei Lincei, 35 (1963), 152-157. 


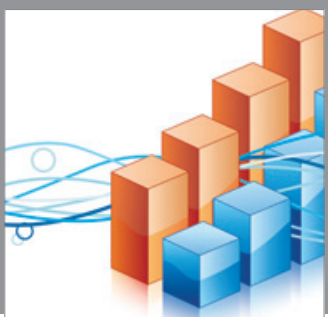

Advances in

Operations Research

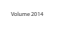

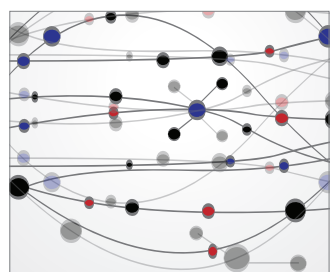

\section{The Scientific} World Journal
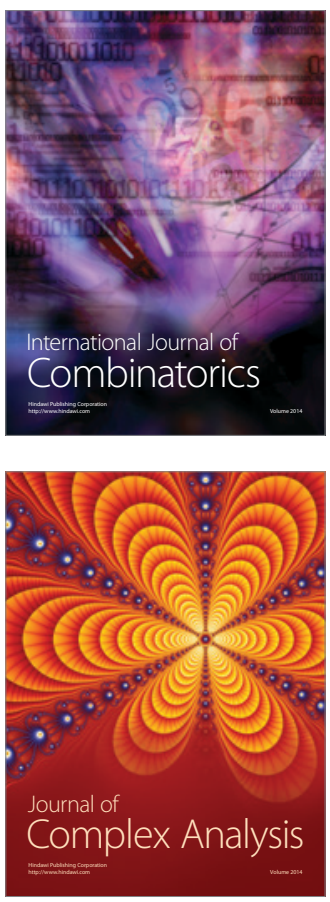

International Journal of

Mathematics and

Mathematical

Sciences
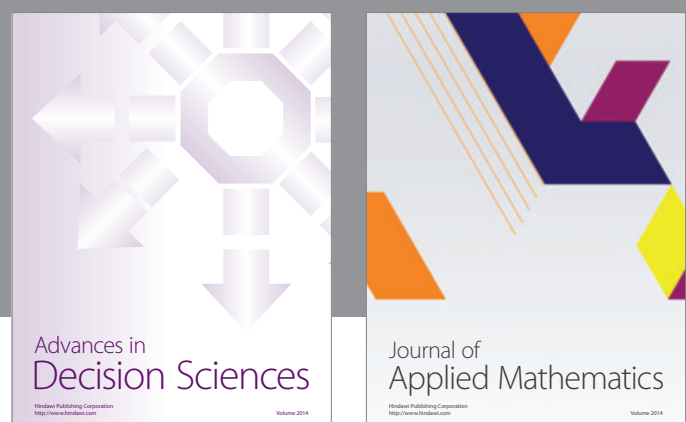

Journal of

Applied Mathematics
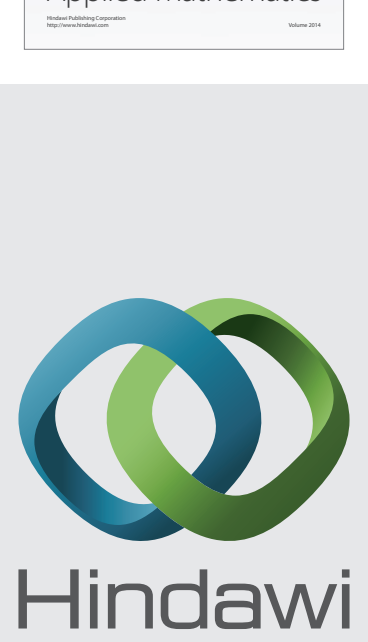

Submit your manuscripts at http://www.hindawi.com
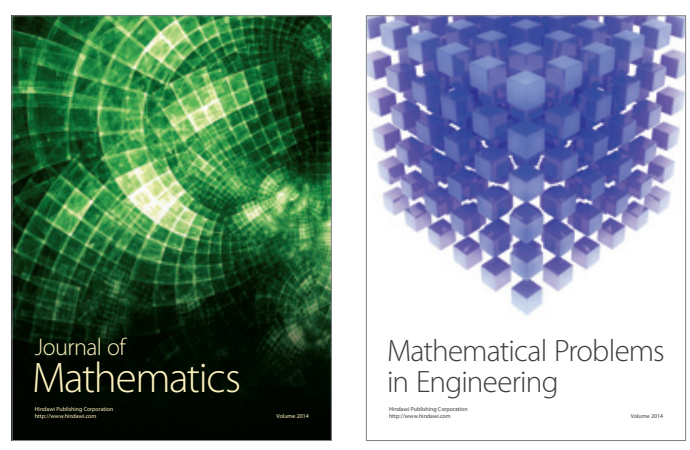

Mathematical Problems in Engineering
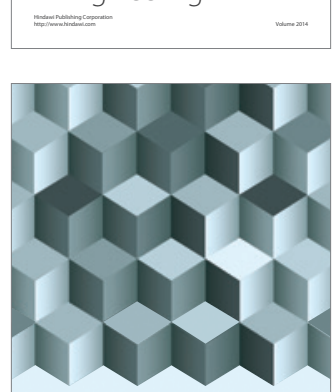

Journal of

Function Spaces
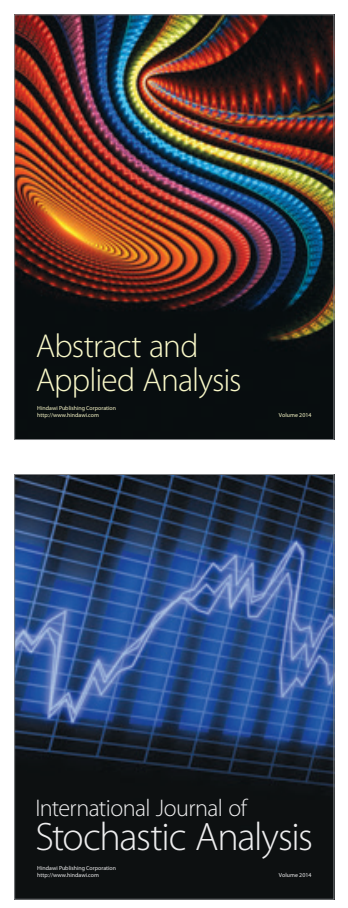

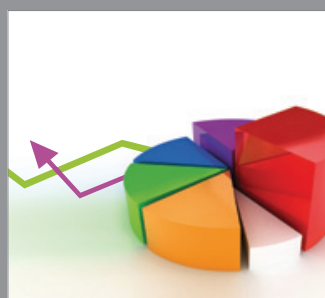

ournal of

Probability and Statistics

Promensencen
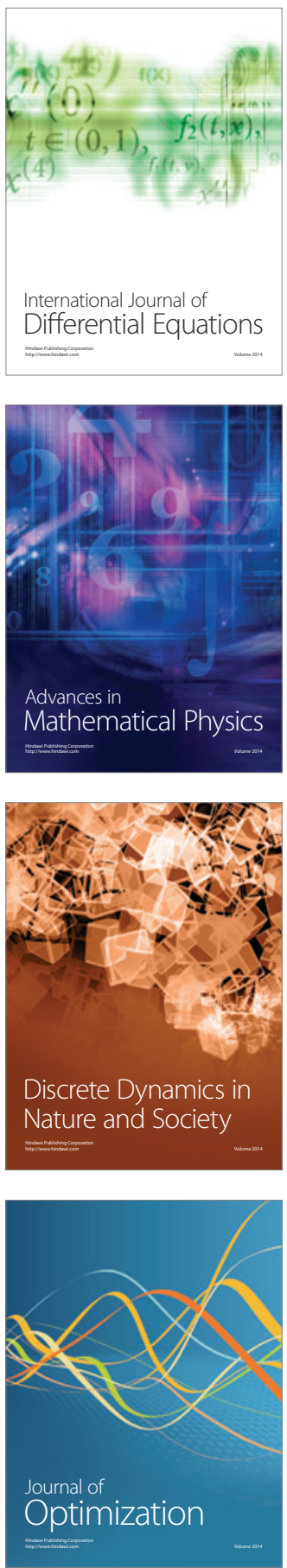